\title{
Design of Collusion-Resistant Fingerprinting Systems: Review and New Results (Invited Paper)
}

\author{
C.-C. Jay Kuo \\ University of Southern California
}

To protect the copyright of a media file, one idea is to develop a traitor tracing system that identifies its unauthorized distribution or usage by tracing fingerprints of illegal users (i.e., traitors). There exists a simple yet effective attack that can break a naive traitor tracing system easily known as the collusion attack. The design of a fingerprinting system that is robust against collusion attacks has been intensively studied in the last decade. A review of previous work in this field will be given. Most previous work deals with collusion attacks with equal weights or its variants (e.g., the cascade of several collusion attacks with equal weights). Consequently, the weight of each colluder is a constant throughout the collusion process. The collusion attack on continuous media such as audio and video with time-varying weights is simple to implement. However, we are not aware of any effective solution to this type of attacks. To address this problem, we first show that this can be formulated as a multi-user detection problem in a wireless communication system with a time-varying channel response. Being inspired by the multi-carrier code division multiaccess (MC-CDMA) technique, we propose a new fingerprinting system that consists of the following modules: 1) codeword generation with a multi-carrier approach, 2) colluder weight estimation, 3) advanced message symbol detection. We construct hiding codes with code spreading followed by multi-carrier modulation. For colluder weight estimation, we show that the colluder weight estimation is analogous to channel response estimation, which can be solved by inserting pilot signals in the embedded fingerprint. As to advanced message symbol detection, we replace the traditional correlation-based detector with the maximal ratio combining (MRC) detector and the parallel interference cancellation (PIC) multiuser detector. The superior performance of the proposed fingerprinting system in terms of user/colluder capacity and the bit error probability (BEP) of symbol detection is demonstrated by representative examples. 